\title{
Pandrug Resistance (PDR), Extensive Drug Resistance (XDR), and Multidrug Resistance (MDR) among Gram-Negative Bacilli
}

\author{
Md. Rafiqul Islam \\ ${ }^{1}$ Professor \& Head, Department of Microbiology, Shaheed Suhrawardy Medical College, Sher-E-Bangla Nagar, Dhaka, Bangladesh;

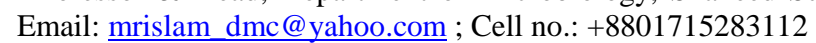

In a recent commentary, Paterson and Doi ${ }^{1}$ addressed the need for universal definitions for the various degrees of antimicrobial resistance among gramnegative bacilli. In light of the recent evidence suggesting that polymyxins, a "rediscovered" category of antimicrobial agents ${ }^{2}$, and tigecycline, a new drug $^{3}$, constitute valuable additions to the clinician's armamentarium against highly resistant gram-negative bacteria, these authors proposed the term "extreme drug resistance" (XDR) to signify resistance of pathogens that are gram negative to all potentially effective antibiotics. They also narrowed the meaning of the already-used term "panresistance" to refer to pathogens that are specifically resistant to 7 antimicrobial agents (cefepime, ceftazidime, imipenem, meropenem, piperacillin-tazobactam, ciprofloxacin, and levofloxacin). Although we welcome the authors' endeavor to suggest terms related to bacterial drug resistance, we would like to express our reservations regarding the proposed definitions and our consideration that the attribution of improper meaning to the aforementioned terms may increase the relevant confusion observed in the medical literature ${ }^{4}$, with potentially important implications for researchers, health care professionals, or even the public. The prefix "pan-" has its origin in the ancient Greek language, meaning "all" or "whole." With this meaning, it is used in common English $^{5}$ and other languages. Of importance, it has been used for the generation of numerous combined biomedical terms, to denote the inclusion of all parts or aspects of an entity ${ }^{6}$. In this respect, the term "panresistance" or "pandrug resistance" (PDR) cannot be interpreted in a sense other than signifying resistance to all antibiotics ${ }^{4,7-8}$. In regard to the term "extreme drug resistance," it was first used in the field of oncology/hematology to designate tumor cell colonies that exhibit significantly decreased responsiveness in vitro to a studied chemotherapeutic agent ${ }^{9}$. In the field of infectious diseases, "XDR" has been used to abbreviate "extensively drug resistant" Mycobacterium tuberculosis, defined by resistance to both rifampin and isoniazid, as well as to fluoroquinolones plus an injectable agent ${ }^{10}$. Presumably, Paterson and $\mathrm{Doi}^{1}$ introduced the term "extreme drug resistance" for gram-negative bacteria to designate a wider resistance pattern than "extensive drug resistance." Still, semantically, "extreme" cannot be more inclusive than "pan-," meaning all. It should also be noted that, in the proposed definitions ${ }^{1}$, a category of highly resistant pathogens, with resistance patterns intermediate to those described in the authors' commentary by the terms "panresistance" and "extreme drug resistance," remains unclassified. The inevitable need to characterize these pathogens with either 1 of the 2 or other terms would eventually generate further confusion. In fact, apart from the term pandrug resistance, notable diversity has been observed as well in the medical literature regarding the use of the term "multidrug resistance" (MDR), which has rather arbitrarily been used to denote resistance of gram-negative pathogens to a varying number of antimicrobial agents ${ }^{4,11}$.

In our view, when all these issues are considered, the terms "pandrug resistance," "extensive drug resistance," and "multidrug resistance" should designate, respectively, resistance of a pathogen to all, resistance to all but 1 or 2 , and resistance to 3 classes of antimicrobial agents, among those that are available at the time of use of the definition and in most parts of the world and that are regarded as potentially effective against the respective pathogen. 
We can advocate the term "extensive drug resistance" rather than "extreme drug resistance" to avoid confusion with the different meaning of the latter term in the context of neoplastic diseases? Furthermore, the definitions of PDR and extensive drug resistance, as we herein state, will retain their semantic properties in time, without the need of introduction of new terms in cases in which more therapeutic options against highly resistant bacteria become available. In addition, because these definitions respect the etymology of the terms used, they are expected to correspond to the use of the same terms in other fields of infectious diseases or in medicine in general. [Bangladesh Journal of Infectious Diseases, 2014;1(2):22-23]

Cited as: Islam MR. Pandrug Resistance (PDR), Extensive Drug Resistance (XDR), and Multidrug Resistance (MDR) among Gram-Negative Bacilli. Bangladesh J Infect Dis, 2014;1(2):2223]

\section{References}

1. Paterson DL, Doi Y. A step closer to extreme drug resistance (XDR) in gram-negative bacilli. Clin Infect Dis 2007; 45:1179-81

2. Falagas ME, Kasiakou SK. Colistin: the revival of polymyxins for the management of multidrug-resistant gramnegative bacterial infections. Clin Infect Dis 2005; 40:133341
3. Hoban DJ, Bouchillon SK, Dowzicky MJ. Antimicrobial susceptibility of extended spectrum b-lactamase producers and multidrug resistant Acinetobacter baumannii throughout the United States and comparative in vitro activity of tigecycline, a new glycylcycline antimicrobial. Diagn Microbiol Infect Dis 2007; 57:423-8

4. Falagas ME, Koletsi PK, Bliziotis IA. The diversity of definitions of multidrug-resistant (MDR) and pandrugresistant (PDR) Acinetobacter baumannii and Pseudomonas aeruginosa. J Med Microbiol 2006; 55:1619-29

5. Merriam-Webster Online Dictionary. 2005. Available at: http://www.merriam-webster.com/. Accessed 10 October 2007

6. Dorland's Illustrated Medical Dictionary, $30^{\text {th }}$ ed. Philadelphia: WB Saunders, 2003

7. Falagas ME, Bliziotis IA, Kasiakou SK, Samonis G, Athanassopoulou P, Michalopoulos A. Outcome of infections due to pandrug resistant (PDR) gram-negative bacteria. BMC Infect Dis 2005; 5:24

8. Falagas ME, Kasiakou SK. Correct use of the term "pandrug-resistant" (PDR) gram-negative bacteria. Clin Microbiol Infect 2005; 11:1049-50

9. Kern DH, Weisenthal LM. Highly specific prediction of antineoplastic drug resistance with an in vitro assay using suprapharmacologic drug exposures. J Natl Cancer Inst 1990; 82:582-8

10. Extensively drug-resistant tuberculosis (XDRTB): recommendations for prevention and control. Wkly Epidemiol Rec 2006; 81:430-2

11. Paterson DL. The epidemiological profile of infections with multidrug-resistant Pseudomonas aeruginosa and Acinetobacter species. Clin Infect Dis 2006; 43(Suppl 2):438 